\title{
A BOUNDARY VALUE PROBLEM IN THE CALCULUS OF VARIATIONS*
}

BY G. A. BLISS

1. Introduction. It is well known that the necessary condition of Jacobi for a problem with fixed end-points in the calculus of variations is closely related to a certain boundary value problem associated with Jacobi's differential equation. $\dagger$ If Jacobi's condition is satisfied then the smallest value $\lambda_{1}$ of the parameter $\lambda$ of the boundary value problem, for which that problem has a solution, must satisfy the inequality $\lambda_{1} \geqq 0$, and conversely. In the following pages it is proposed to deduce a similar relationship for problems of the calculus of variations with variable end-points. The corresponding boundary value problem has end conditions of a more general type than those which arise when the end-points of the original calculus of variations problem are fixed. The existence of the smallest value $\lambda_{1}$ is established by methods of the calculus of variations, in particular by means of a theorem analogous to a well known theorem of Osgood $t$ It seems likely that a complete theory of self-adjoint boundary value problems for ordinary differential equations, with end conditions of a very general type, can be deduced from theorems already well known in the calculus of variations. So far as I know this has never been done, though many significant relationships have of course been pointed out. $\S$

2. The Calculus of Variations Problem and its Second Variation. Let $C_{1}$ and $C_{2}$ be two arcs with the parametric equations

* Presented to the Society, April 14, 1922.

$\dagger$ See, for example, Lovitt, Linear Integral Equations, p. 207.

¥ Transactions of this Society, vol. 2 (1901), p. 273.

$\S$ See, for example, Richardson, Mathematische Annalen, vol. 68 (1910), p. 279; Plancherel, Bulletin des Sciences Mathématiques, vol. 47 (1923), p. 376. 


$$
x=X_{i}(u), \quad y=Y_{i}(u),
$$

and let $E$ be an arc

$$
y=y(x) \quad\left(x_{1} \leqq x \leqq x_{2}\right)
$$

intersecting them, respectively, in points 1 and 2 , as shown in the accompanying figure. We suppose that the functions defining $C_{1}$ and $C_{2}$ have continuous first and second deriva-

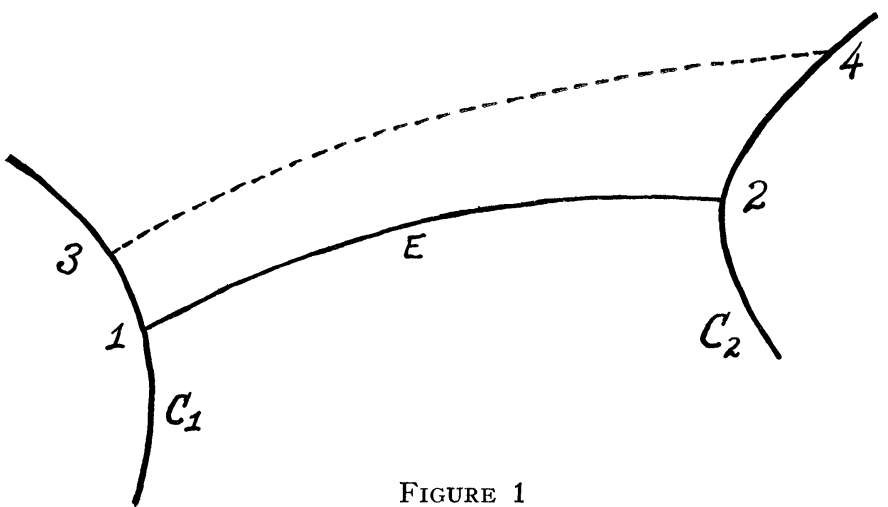

FiguRe 1

tives and that these arcs have no singular points. The arc $E$ is continuous and consists of a finite number of pieces each of which has a continuously turning tangent.

Consider now an integral

$$
I=\int_{x_{1}}^{x_{2}} f\left(x, y, y^{\prime}\right) d x
$$

whose integrand function $f\left(x, y, y^{\prime}\right)$ has continuous partial derivatives up to and including those of the fourth order for all sets of values $\left(x, y, y^{\prime}\right)$ in a neighborhood of those on $E$. The arcs which have continuity properties similar to those of $E$ and whose elements all lie in this neighborhood may be called admissible arcs. The problem before us is to discuss the necessary conditions which $E$ must satisfy if it minimizes $I$ in the class of admissible arcs joining $C_{1}$ and $C_{2}$.

It is well known that an equation $f_{y^{\prime}}=\int_{a}^{x} f_{y} d x+c$ must be satisfied by $E$, from which it follows that the function $f_{y^{\prime}}$ is 
necessarily continuous, when considered as a function of $x$ along $E$, even at the corners of $E$, and that the Euler equation

$$
\frac{d}{d x} f_{y^{\prime}}=f_{y}
$$

is satisfied on every sub-arc of $E$ on which the tangent turns continuously. Furthermore at the points 1 and 2 the transversality condition

$$
f\left(x, y, y^{\prime}\right) X^{\prime}+\left(Y^{\prime}-y^{\prime} X^{\prime}\right) f_{y^{\prime}}\left(x, y, y^{\prime}\right)=0
$$

must be satisfied by the element $\left(x, y, y^{\prime}\right)$ of $E$ and the direction $X^{\prime}: Y^{\prime}$ of the arc $C_{1}$ or $C_{2} . \dagger$ We assume, as is customary for problems with variable end-points in the calculus of variations, that the value of $f$ at the points 1 and 2 on $E$ is different from zero. Since $C_{1}$ and $C_{2}$ have no singular points it follows from this assumption and equation (2) that the difference $Y^{\prime}-y^{\prime} X^{\prime}$ is not zero at the points 1 and 2 , or in other words that $E$ is not tangent to either $C_{1}$ or $C_{2}$.

In the discussion of further necessary conditions for a minimum we may agree to limit ourselves to $\operatorname{arcs} E$ for which the function $\dot{y}(x)$ has continuous first and second derivatives on an interval containing $x_{1} x_{2}$, and we may consider as usual a one-parameter family of arcs of the form

$$
y=y(x)+a \eta(x)=y(x, a)
$$

where $\eta$ has continuity properties like those just prescribed for $y(x)$ but is otherwise arbitrary. The equations

$$
Y_{1}\left(u_{3}\right)-y\left(X_{1}\left(u_{3}\right), a\right)=0, \quad Y_{2}\left(u_{4}\right)-y\left(X_{2}\left(u_{4}\right), a\right)=0
$$

determine the parameter values $u_{3}$ and $u_{4}$ where the arc (3) intersects $C_{1}$ and $C_{2}$, respectively, as shown in Figure 1. The former, for example, has a solution $\left(u_{3}, a\right)=\left(u_{1}, 0\right)$, corresponding to the point 1 of the figure, at which the derivative of its first member with respect to $u_{3}$ is

$$
Y_{1}^{\prime}\left(u_{1}\right)-y_{x}\left(X_{1}^{\prime}\left(u_{1}\right), 0\right) X_{1}^{\prime}\left(u_{1}\right)=Y_{1}^{\prime}\left(u_{1}\right)-y^{\prime}\left(x_{1}\right) X_{1}^{\prime}\left(u_{1}\right) \neq 0 .
$$

* Bliss, Calculus of Variations, p. 130.

$\dagger$ Bliss, loc. cit., p. 167. 
It follows from well known implicit function theorems that the first equation (4) has a unique solution $u_{3}(a)$ with continuous first and second derivatives near $a=0$ and with the initial value $u_{3}(0)=u_{1}$. The second has a similar solution $u_{4}(a)$ with initial value $u_{4}(0)=u_{2}$. The derivatives at $a=0$ of these two functions are both defined by the equation

$$
\frac{d u}{d a}=\frac{y_{a}(X, 0)}{Y^{\prime}-y_{x}(X, 0) X^{\prime}}=\frac{\eta(x)}{Y^{\prime}-y^{\prime}(X) X^{\prime}}
$$

when suitable subscripts are attached to $X$ and $Y$, and when the arguments $u_{1}$ and $u_{2}$, respectively, are substituted. The coordinates of the intersection points 3 and 4 are also functions of $a$ defined by the equations

$$
\begin{array}{ll}
x_{3}(a)=X_{1}\left(u_{3}(a)\right), & y_{3}(a)=y\left(x_{3}(a), a\right)=Y_{1}\left(u_{3}(a)\right), \\
x_{4}(a)=X_{2}\left(u_{4}(a)\right), & y_{4}(a)=y\left(x_{4}(a), a\right)=Y_{2}\left(u_{4}(a)\right),
\end{array}
$$

and their derivatives satisfy the relations

$$
\left\{\begin{array}{l}
\frac{d x}{d a}=X^{\prime} \frac{d u}{d a}, \quad \frac{d^{2} x}{d a^{2}}=X^{\prime} \frac{d^{2} u}{d a^{2}}+X^{\prime \prime}\left(\frac{d u}{d a}\right)^{2} \\
\frac{d y}{d a}=y_{x} \frac{d x}{d a}+y_{a}=Y^{\prime} \frac{d u}{d a}, \\
\frac{d^{2} y}{d a^{2}}=y_{x} \frac{d^{2} x}{d a^{2}}+y_{x x}\left(\frac{d x}{d a}\right)^{2}+2 y_{x a} \frac{d x}{d a}=Y^{\prime} \frac{d^{2} u}{d a^{2}}+Y^{\prime \prime}\left(\frac{d u}{d a}\right)^{2}
\end{array}\right.
$$

If the arc $E$ minimizes $I$ as described above the function

$$
I(a)=\int_{x_{3}}^{x_{4}} f\left(x, y(x, a), y_{x}(x, a)\right) d x
$$

taken along the arc (3) from its intersection 3 with $C_{1}$ to its intersection 4 with $C_{2}$ must have a minimum at the value $a=0$, and its derivatives must satisfy the conditions $I^{\prime}(0)=0$, $I^{\prime \prime}(0) \geqq 0$. The values of these derivatives are readily found to be

$$
I^{\prime}(a)=\left.f \frac{d x}{d a}\right|_{3} ^{4}+\int_{x_{3}}^{x_{4}}\left(f_{y} y_{a}+f_{y^{\prime}} y_{x a}\right) d x,
$$




$$
\begin{aligned}
I^{\prime \prime}(a) & =f \frac{d^{2} x}{d a^{2}}+\left(f_{x}+f_{y} y_{x}+f_{y^{\prime}} y_{x x}\right)\left(\frac{d x}{d a}\right)^{2} \\
+ & \left.2\left(f_{y} y_{a}+f_{y^{\prime}} y_{x a}\right) \frac{d x}{d a}\right|_{3} ^{4}+\int_{x_{3}}^{x_{4}} 2 \omega\left(x, y_{a}, y_{a x}\right) d x
\end{aligned}
$$

where $2 \omega\left(x, \eta, \eta^{\prime}\right)=f_{y y} \eta^{2}+2 f_{y y^{\prime}} \eta \eta^{\prime}+f_{y^{\prime} y^{\prime}} \eta^{\prime 2}$. When $a=0$, the first of these vanishes, as may be shown by integrating the second term of the integrand by parts and applying Euler's equation (1) and the transversality conditions (2). With the help of the last equation (6) the second derivative at $a=0$ takes the form

$$
\begin{gathered}
I^{\prime \prime}(0)=\left(f-y_{x} f_{y^{\prime}}\right) \frac{d^{2} x}{d a^{2}}+f_{y^{\prime}} \frac{d^{2} y}{d a^{2}}+\left(f_{x}+y_{x} f_{y}\right)\left(\frac{d x}{d a}\right)^{2} \\
+\left.2 f_{y} y_{a} \frac{d x}{d a}\right|_{1} ^{2}+\int_{x_{1}}^{x_{2}} 2 \omega\left(x, y_{a}, y_{a x}\right) d x .
\end{gathered}
$$

When the partial derivatives $y_{a}=\eta$ and the total derivatives of $x$ and $y$ with respect to $a$ are substituted from equations (6) and (5) it is found after some computation that this derivative has the value

$$
I^{\prime \prime}(0)=\left.L \eta^{2}\right|_{1} ^{2}+\int_{x_{1}}^{x_{2}} 2 \omega\left(x, \eta, \eta^{\prime}\right) d x
$$

where $L$ is defined by the equations

$$
\begin{gathered}
L=\frac{M}{r}+N, \quad M=\left[X^{\prime} f_{y^{\prime}}-Y^{\prime}\left(f-y^{\prime} f_{y^{\prime}}\right)\right] \frac{\sqrt{X^{\prime 2}+Y^{\prime 2}}}{\left(Y^{\prime}-y^{\prime} X^{\prime}\right)^{2}}, \\
N=\left[\left(f_{x}+f_{y} y^{\prime}\right) X^{\prime}+2 f_{y}\left(Y^{\prime}-y^{\prime} X^{\prime}\right)\right] \frac{X^{\prime}}{\left(Y^{\prime}-y^{\prime} X^{\prime}\right)^{2}},
\end{gathered}
$$

in which $r$ is the radius of curvature of $C_{1}$ or $C_{2}$. The only term which might cause difficulty in this computation is the term $M / r$ in $L$. To find it we note with the help of equations (6) that

$$
\begin{gathered}
\left(f-y^{\prime} f_{y^{\prime}}\right) \frac{d^{2} x}{d a^{2}}+f_{y^{\prime}} \frac{d^{2} y}{d a^{2}}=\left[\left(f-y^{\prime} f_{y^{\prime}}\right) X^{\prime}+f_{y^{\prime}} Y^{\prime}\right] \frac{d^{2} u}{d a^{2}} \\
+\left[\left(f-y^{\prime} f_{y^{\prime}}\right) X^{\prime \prime}+f_{y^{\prime}} Y^{\prime \prime}\right]\left(\frac{d u}{d a}\right)^{2} .
\end{gathered}
$$


The coefficient of $d^{2} u / d a^{2}$ in this expression vanishes at 1 and 2 on account of the transversality condition (2), and it follows from the same condition that at those points

$$
f-y^{\prime} f_{y^{\prime}}=-k Y^{\prime}, \quad f_{y^{\prime}}=k X^{\prime}, \quad k=\frac{X^{\prime} f_{y^{\prime}}-Y^{\prime}\left(f-y^{\prime} f_{y^{\prime}}\right)}{X^{\prime 2}+Y^{\prime 2}} .
$$

With the help of the value (5) for $d u / d a$ we now find that the value of the expression (8) is $(M / r) \eta^{2}$. The other terms in the expression for $I^{\prime \prime}(0)$ give no trouble, and we may summarize the results of this section as follows.

Theorem 1. If the arc $E$ is to minimize the integral $I$ in a class of neighboring arcs joining the curves $C_{1}$ and $C_{2}$, as described above, then the expression (7) for the second variation $I^{\prime \prime}(0)$ must be $\geqq 0$ for every function $\eta(x)$ having continuous first and second derivatives on the interval $x_{1} x_{2}$.

One can infer readily that $I^{\prime \prime}(0)$ must also be $\geqq 0$ for all continuous functions $\eta(x)$ on $x_{1} x_{2}$ which have continuous first derivatives except possibly at a finite number of corners. This can be done by modifying suitably the analysis of the preceding paragraphs, or by showing that every function $\eta(x)$ of this character can be approximated by a polynomial giving $I^{\prime \prime}(0)$ a value near to that for $\eta(x){ }^{*}$

3. The Problem of Minimizing the Second Variation. Let us consider in this section the problem of determining when an expression of the form

$$
I_{2}(\eta)=\int_{x_{1}}^{x_{2}} 2 \Omega\left(x, \eta, \eta^{\prime}\right) d x
$$

is $\geqq 0$ in the class $H$ of functions $\eta(x)$ continuous on $x_{1} x_{2}$ and having continuous derivatives except possibly at a finite number of corners. The integrand $2 \Omega$ is understood to be a quadratic form

$$
2 \Omega\left(x, \eta, \eta^{\prime}\right)=P(x) \eta^{2}+2 Q(x) \eta \eta^{\prime}+R(x) \eta^{\prime 2}
$$

with $R>0$ on $x_{1} x_{2}$ and with $P, Q, R$ having continuous first

\footnotetext{
* Hadamard, Calcul des Variations, pp. 51-54.
} 
derivatives on this interval. The second variation (7) is easily seen to be an integral of the type $I_{2}(\eta)$ if we set

$$
2 \Omega=\frac{d}{d x} L \eta^{2}+f_{y y} \eta^{2}+2 f_{y y^{\prime}} \eta \eta^{\prime}+f_{y^{\prime} y^{\prime}} \eta^{\prime 2},
$$

where $L$ is a function of $x$ with continuous second derivatives on $x_{1} x_{2}$ and with $L_{1}$ and $L_{2}$ as initial and end-values on this interval.

The problem of minimizing $I_{2}(\eta)$ in the class $H$ of functions $\eta(x)$ is that of finding a curve in the $x \eta$-plane joining the two ordinates $x=x_{1}$ and $x=x_{2}$ and minimizing $I_{2}(\eta)$. It is therefore of the same type as the original problem of the last section in the $x y$-plane, and it is clear that a minimizing curve for the $x \eta$-problem must satisfy an equation

$$
\Omega_{\eta^{\prime}}=\int_{x_{1}}^{x} \Omega_{\eta} d x+c
$$

and the transversality conditions

$$
\left.\Omega_{\eta^{\prime}}\right|^{1}=0,\left.\quad \Omega_{\eta^{\prime}}\right|^{2}=0 \text {. }
$$

If we substitute the derivatives of the quadratic form $\Omega$ in these three equations we find from the first that a minimizing function $\eta(x)$ must have continuous first and second derivatives and satisfy the Jacobi equation

$$
\Omega_{\eta}-\frac{d}{d x} \Omega_{\eta^{\prime}}=\left(P-Q^{\prime}\right) \eta-R^{\prime} \eta^{\prime}-R \eta^{\prime \prime}=0
$$

where $\Omega$ is defined by the equation preceding (9). This is identical with the Jacobi equation deduced from the quadratic form $2 \omega$ in the second variation (7) when $2 \Omega$ has the value (9), as one readily verifies. The equations (10) show that a minimizing function must further satisfy the boundary conditions

$$
Q_{1} \eta\left(x_{1}\right)+R_{1} \eta^{\prime}\left(x_{1}\right)=0, \quad Q_{2} \eta\left(x_{2}\right)+R_{2} \eta^{\prime}\left(x_{2}\right)=0,
$$

where the numerical subscripts designate the values of $Q(x), R(x)$ at $x_{1}$ or $x_{2}$. One can readily interpret these conditions in terms of the coefficients of the quadratic form $2 \omega$ 
and the constants $L_{1}$ and $L_{2}$ of the second variation when $2 \Omega$ has the value (9). We have then the following result.

Theorem 2. A function $\eta(x)$ which minimizes $I_{2}(\eta)$ in the class $H$ must have continuous first and second derivatives and be a solution of the boundary value problem

$$
J(\eta)=0, \quad Q_{1} \eta\left(x_{1}\right)+R_{1} \eta^{\prime}\left(x_{1}\right)=Q_{2} \eta\left(x_{2}\right)+R_{2} \eta^{\prime}\left(x_{2}\right)=0,
$$

where $J(\eta)$ is a symbol for the first member of the Jacobi equation (11).

We shall need presently the following lemma.

LEMma 1. If $u(x)$ is a solution of the equation $J(u)=0$ and is different from zero on $x_{1} x_{2}$ then for every $\eta(x)$ in the class $H$

$$
\int_{x_{1}}^{x_{2}} 2 \Omega d x=\left.\frac{\eta^{2}}{u} \Omega_{u^{\prime}}\right|_{1} ^{2}+\int_{x_{1}}^{x_{2}} R(x)\left(\eta^{\prime \prime}-\frac{\eta}{u} u^{\prime}\right)^{2} d x
$$

where $\Omega_{u^{\prime}}=\Omega_{\eta^{\prime}}\left(x, u, u^{\prime}\right)$.

To prove this consider the function $a(x)$ and its derivative determined by the equations

$$
\eta(x)=a(x) u(x), \quad \eta^{\prime}=a u^{\prime}+a^{\prime} u .
$$

With the help of Taylor's formula and the equation $J(u)=0$ we see that

$$
\begin{aligned}
2 \Omega\left(x, \eta, \eta^{\prime}\right) & =2 \Omega\left(x, a u, a u^{\prime}\right)+2 a^{\prime} u \Omega_{\eta^{\prime}}\left(x, a u, a u^{\prime}\right)+R a^{\prime 2} u^{2} \\
& =a^{2}\left(u \Omega_{u}+u^{\prime} \Omega_{u^{\prime}}\right)+2 a a^{\prime} u \Omega_{u^{\prime}}+R a^{\prime 2} u^{2} \\
& =\frac{d}{d x} a^{2} u \Omega_{u^{\prime}}+R\left(\eta^{\prime}-a u^{\prime}\right)^{2} \\
& =\frac{d}{d x} \frac{\eta^{2}}{u} \Omega_{u^{\prime}}+R\left(\eta^{\prime}-\frac{\eta}{u} u^{\prime}\right)^{2} .
\end{aligned}
$$

An integration gives at once the formula of the lemma.

Theorem 3. A necessary and sufficient condition that the relation $I_{2}(\eta) \geqq 0$ shall hold for every function $\eta(x)$ of the class $H$ is that the two solutions $u_{1}(x), u_{2}(x)$ of the equation $J(u)=0$ defined by the conditions 


$$
\begin{array}{ll}
u_{1}\left(x_{1}\right)=R_{1}, & u_{1}^{\prime}\left(x_{1}\right)=-Q_{1}, \\
u_{2}\left(x_{2}\right)=R_{2}, & u_{2}^{\prime}\left(x_{2}\right)=-Q_{2},
\end{array}
$$

be positive and have

$$
u_{2} u_{1}^{\prime}-u_{1} u_{2}^{\prime}>0
$$

on the interval $x_{1} x_{2}$.

To prove the necessity we note first that for every solution $u(x)$ of the equation $J(u)=0$ we have

$$
2 \Omega\left(x, u, u^{\prime}\right)=u \Omega_{u}+u^{\prime} \Omega_{u^{\prime}}=\frac{d}{d x} u \Omega_{u^{\prime}} .
$$

The function $u_{1}(x)$ is positive at $x=x_{1}$ and can not vanish at any value $\xi$ between $x_{1}$ and $x_{2}$. Otherwise we could define $\eta(x)$ by the equations

$$
\begin{aligned}
& \eta(x)=u_{1}(x) \quad \text { for } \quad x_{1} \leqq x \leqq \xi, \\
& =0 \text { for } \xi \leqq x \leqq x_{2},
\end{aligned}
$$

and for this function we should find with the help of (15) the value

$$
I_{2}(\eta)=\left.u_{1} \Omega_{u_{1}^{\prime}}\right|_{x_{1}} ^{\xi}=0
$$

since $\Omega_{u_{1}^{\prime}}$ vanishes at $x_{1}$ and $u_{1}$ at $\xi$. But this function $\eta(x)$ would have a discontinuous derivative at $x=\xi$ since a solution $u_{1}(x)$ of $J(u)=0$ can vanish with its derivative only when identically zero. Hence $\eta(x)$ could not minimize $I_{2}(\eta)$ and there would be $\eta$ 's giving $I_{2}(\eta)$ negative values. In the same manner we may prove that $u_{2}(x)$ does not vanish between $x_{1}$ and $x_{2}$ when the values of $I_{2}(\eta)$ are all $\geqq 0$ in $H$.

The equation

$$
0=u_{1} J\left(u_{2}\right)-u_{2} J\left(u_{1}\right)=\frac{d}{d x} R\left(u_{2} u_{1}^{\prime}-u_{1} u_{2}^{\prime}\right)
$$

shows that

$$
R\left(u_{2} u_{1}^{\prime}-u_{1} u_{2}^{\prime}\right)=c
$$

and hence that the determinant (14) has always the same 
sign or else vanishes identically on $x_{1} x_{2}$. We may define $\eta(x)$ by the equations

$$
\begin{aligned}
& \eta(x)=u_{2}(\xi) u_{1}(x) \quad \text { for } \quad x_{1} \leqq x \leqq \xi, \\
& =u_{1}(\xi) u_{2}(x) \quad \text { for } \quad \xi \leqq x \leqq x_{2},
\end{aligned}
$$

and the value of $I_{2}(\eta)$, with the help of (15), is then

$$
I_{2}(\eta)=u_{1} u_{2}\left(u_{2} \Omega_{u_{1}^{\prime}}-u_{1} \Omega_{u_{2}^{\prime}}\right) \mid \xi=c u_{1}(\xi) u_{2}(\xi)
$$

which would be negative, since $u_{1}$ and $u_{2}$ are both positive between $x_{1}$ and $x_{2}$, if the constant $c$ in equation (16) were negative. Hence we see that the determinant (14) can not be negative when $I_{2}(\eta) \geqq 0$ in $H$.

The function $u_{1}(x)$ can not vanish even at $x=x_{2}$ since if $u_{1}\left(x_{2}\right)$ were zero we should have $u_{1}^{\prime}\left(x_{2}\right)<0$ and the determinant (14) would be negative since $u_{2}\left(x_{2}\right)$ is positive. Similarly $u_{2}(x)$ can not vanish at $x_{1}$.

To prove the sufficiency of the condition of the theorem let $\eta(x)$ be an arbitrary function of the class $H$ and let us apply the formula (12) to $\eta$ and $u_{1}$ on an interval $x_{1} \xi$, and to $\eta$ and $u_{2}$ on the remaining interval $\xi x_{2}$. Then

$$
\begin{aligned}
I_{2}(\eta) & =\frac{\eta^{2}}{u_{1}} \Omega_{u_{1}}-\left.\frac{\eta^{2}}{u_{2}} \Omega_{u_{2}^{\prime}}\right|^{\xi}+\int_{x_{1}}^{x_{2}} R\left(\eta^{\prime}-\frac{\eta}{u} u^{\prime}\right)^{2} d x \\
& =\frac{c \eta^{2}(\xi)}{u_{1}(\xi) u_{2}(\xi)}+\int_{x_{1}}^{x_{2}} R\left(\eta^{\prime}-\frac{\eta}{u} u^{\prime}\right)^{2} d x
\end{aligned}
$$

where $u$ in the last integral is $u_{1}$ on $x_{1} \xi$, and $u_{2}$ on $\xi x_{2}$. The last expression is evidently not negative under the hypothesis of the theorem.

Corollary 1. A necessary and sufficient condition that the relation $I_{2}(\eta)>0$ shall hold for every function of $H$ except $\eta(x) \equiv 0$ is that $u_{1}(x)$ and $u_{2}(x)$ be positive and have

$$
u_{2} u_{1}^{\prime}-u_{1} u_{2}^{\prime}>0
$$

on the interval $x_{1} x_{2}$.

To prove the necessity we observe that the last theorem requires $u_{1}$ and $u_{2}$ to be positive and $u_{2} u_{1}^{\prime}-u_{1} u_{2}^{\prime}$ to be $\geqq 0$ 
on $x_{1} x_{2}$. But if this determinant were zero we should have $u_{1}=k u_{2}$, and then $\eta=u_{1}$ would make $I_{2}(\eta)=0$, as we readily see by applying equations (15) and (13). Hence $I_{2}(\eta)$ would vanish for an $\eta(x) \neq 0$ in $H$ unless the condition (18) is satisfied.

To prove the sufficiency we note that the first term in the expression (17) is certainly positive for some value of $\xi$, under the hypotheses of the theorem, unless $\eta(x) \equiv 0$ on $x_{1} x_{2}$.

Corollary 2. If the condition of the last corollary holds then there is a constant $\delta>0$ such that $I_{2}(\eta)>\delta$ for every function $\eta(x)$ of the class $H$ satisfying the equation

$$
\int_{x_{1}}^{x_{2}} \eta^{2}(x) d x=1
$$

This follows from the formula (17) since for such an $\eta(x)$ there must be at least one point $\xi$ on the interval $x_{1} x_{2}$ where $\eta^{2}(\xi)>1 /\left(x_{2}-x_{1}\right)$. The corollary is analogous to the theorem of Osgood cited on a preceding page.

Let us define $H_{1}$ as the class of all functions of $H$ which satisfy the equation (19). Then it is of interest to note the following theorem.

ThEOREM 4. A function $\eta(x)$ which minimizes $I_{2}(\eta)$ in the class $H_{1}$ is necessarily a solution of the boundary value problem

$$
\begin{gathered}
J(\eta)-\lambda_{\eta}=0, \\
Q_{1} \eta\left(x_{1}\right)+R_{1} \eta^{\prime}\left(x_{1}\right)=Q_{2} \eta\left(x_{2}\right)+R_{2} \eta^{\prime}\left(x_{2}\right)=0 .
\end{gathered}
$$

For suppose that $\eta(x)$ is such a minimizing function and consider the two-parameter family of functions $\eta+a \zeta+a_{1} \zeta_{1}$ where $\zeta$ and $\zeta_{1}$ are two arbitrarily selected functions of $H$. Denote by $I\left(a, a_{1}\right), K\left(a, a_{1}\right)$ the values of $I_{2}$ and the integral in (19) on an arc of this family. The determinant

$$
I_{a}(0,0) K_{a_{1}}(0,0)-I_{a_{1}}(0,0) K_{a}(0,0)
$$

must be zero for every choice of $\zeta$ and $\zeta_{1}$. Otherwise, according to well known implicit function theorems, the equations

$$
I\left(a, a_{1}\right)=I(0,0)+u, \quad K\left(a, a_{1}\right)=1
$$


would have solutions for both positive and negative values of $u$ near $u=0$, and $I(0,0)$ could not be a minimum value of $I\left(a, a_{1}\right)$. Since the determinant vanishes for every choice of $\zeta$ and $\zeta_{1}$ let us fix $\zeta_{1}$ and choose $\lambda$ so that the second of the equations

$$
I_{a}(0,0)-\lambda K_{a}(0,0)=0, \quad I_{a_{1}}(0,0)-\lambda K_{a_{1}}(0,0)=0
$$

is satisfied. Then the first must hold for every $\zeta$ in the class $H$. It has the form

$$
I_{a}(0,0)-\lambda K_{a}(0,0)=\int_{x_{1}}^{x_{2}} 2\left[\Omega_{\eta} \zeta+\Omega_{\eta^{\prime}} \zeta^{\prime}-\lambda \eta \zeta\right] d x=0 .
$$

Since this must be true for every $\zeta$ in $H$ it follows, by the customary integration of the second term of the integrand by parts, that $\eta$ satisfies the conditions (20), which was to be proved.

It is evident that if the expression $I_{2}(\eta)$ is $\geqq 0$ in the class $H$ it will also have this property in the class $H_{1}$, and conversely. We shall in the next section be concerned with the problem of determining the minimum of $I_{2}(\eta)$ in the class $H_{1}$.

4. The Boundary-Value Problem. From the theorems of the preceding section it may be inferred that the solution of the boundary value problem

$$
\begin{gathered}
J(\eta)-\lambda \eta=0, \\
Q_{1} \eta\left(x_{1}\right)+R_{1} \eta^{\prime}\left(x_{1}\right)=Q_{2} \eta\left(x_{2}\right)+R_{2} \eta^{\prime}\left(x_{2}\right)=0
\end{gathered}
$$

is closely related to the problem of determining the minimum of the integral

$$
I_{2}(\eta)=\int_{x_{1}}^{x_{2}} 2 \Omega\left(x \cdot n \cdot n^{\prime}\right) d x
$$

in the class $H_{1}$ of functions $\eta(x)$ satisfying the equation

$$
\int_{x_{1}}^{x_{2}} \eta^{2}(x) d x=1
$$

where $2 \Omega=P(x) \eta^{2}+2 Q(x) \eta \eta^{\prime}+R(x) \eta^{\prime 2}$. It is the purpose of this section to investigate this relationship further.

THEOREM 5. The values of the integral $I_{2}(\eta)$ have a greatest lower bound $\lambda_{1}$ in the class $H_{1}$. 
For we may readily verify that

$$
\begin{aligned}
I_{2}(\eta)-\lambda \int_{x_{1}}^{x_{2}} \eta^{2} d x=\int_{x_{1}}^{x_{2}} & {\left[R\left(\frac{Q}{R} \eta+\eta^{\prime}\right)^{2}\right.} \\
& \left.+\left(P-\frac{Q^{2}}{R}-\lambda\right) \eta^{2}\right] d x .
\end{aligned}
$$

By taking $\lambda$ negative and sufficiently large the coefficient of $\eta^{2}$ in the last integral will be positive on $x_{1} x_{2}$ and the integral itself will be positive for every function $\eta(x)$ in $H$ not identically zero. From the first member of the equation it follows then that $I_{2}(\eta)$ will be greater than $\lambda$ for every function $\eta(x)$ in $H_{1}$.

THEOREM 6. The boundary value problem (20) has a solution for $\lambda=\lambda_{1}$, and this is the smallest value of $\lambda$ for which such a solution exists.

It is provable readily that no solution of the boundary value problem exists for a value $\lambda<\lambda_{1}$. Such a solution would give $I_{2}(\eta)$ the value

$$
\begin{aligned}
I_{2}(\eta)= & \int_{x_{1}}^{x_{2}}\left(\eta \Omega_{\eta}+\eta^{\prime} \Omega_{\eta^{\prime}}\right) d x \\
& =\left.\eta \Omega_{\eta^{\prime}}\right|_{1} ^{2}+\int_{x_{1}}^{x_{2}} \eta J(\eta) d x=\lambda \int_{x_{1}}^{x_{2}} \eta^{2} d x,
\end{aligned}
$$

since $\Omega_{\eta^{\prime}}$ vanishes at $x_{1}$ and $x_{2}$ and $J(\eta)=\lambda \eta$. By multiplying $\eta(x)$ by a suitable constant a function of $H_{1}$ would be found giving $I_{2}(\eta)$ the value $\lambda$ which is less than the minimum $\lambda_{1}$.

The expression

$$
I_{2}(\eta)-\lambda \int_{x_{1}}^{x_{2}} \eta^{2} d x
$$

is always $\geqq 0$ in $H_{1}$ when $\lambda=\lambda_{1}$, and hence also in $H$, as one easily verifies. By Theorem 3 of the preceding section it follows that the solutions $u_{1}(x, \lambda), u_{2}(x, \lambda)$ of the equation $J(u)-\lambda u=0$, determined by the conditions (13), must for $\lambda=\lambda_{1}$ be positive and satisfy the relation

$$
u_{2} u_{1}{ }^{\prime}-u_{1} u_{2}{ }^{\prime} \geqq 0
$$


on the interval $x_{1} x_{2}$. If the inequality were true then Corollary 2 of Theorem 3 shows that in the class $H_{1}$ the expression (22) for $\lambda=\lambda_{1}$ would always exceed a constant $\delta>0$, or in other words that $I_{2}(\eta)>\lambda_{1}+\delta$ in $H_{1}$. But this is impossible when $\lambda_{1}$ is the minimum described above. Hence the equality sign in the relation (23) must hold. But in that case $u_{1}=k u_{2}$ and $u_{1}$ is therefore a solution of the boundary value problem (20).

Corollary 1. A necessary and sufficient condition that the relation $I_{2}(\eta) \geqq 0$ holds for every function $\eta(x)$ of the class $H$ is that $\lambda_{1} \geqq 0$ where $\lambda_{1}$ is the smallest value for which the boundary value problem (20) has a solution. A necessary and sufficient condition that $I_{2}(\eta)>0$ for every function $\eta(x)$ in $H$ except $\eta(x) \equiv 0$ is $\lambda_{1}>0$.

This conclusion is an immediate consequence of the fact that $\lambda_{1}$ is the minimum of the values of $I_{2}(\eta)$ in the class $H_{1}$.

Corollary 2. Let $E$ be an arc, as described in Section 1, which gives the integral I there considered the value $I(E)$, and suppose that along $E$ the derivative $f_{y^{\prime} y^{\prime}}$ is positive. Then a necessary condition for $I(E)$ to be a minimum is that $\lambda_{1} \geqq 0$ where $\lambda_{1}$ is the smallest value of $\lambda$ for which the boundary value problem (20) associated with the second variation has a solution.

It should be remarked that the necessary condition $\lambda_{1} \geqq 0$ is equivalent to the well known analog of Jacobi's condition for this calculus of variations problem.* The focal points of the curves $C_{1}$ and $C_{2}$ on $E$ are determined by the roots of $u_{1}(x, 0)$ and $u_{2}(x, 0)$, as one readily verifies. The condition $\lambda_{1} \geqq 0$ implies that the integral $I_{2}(\eta)$ is $\geqq 0$ for all functions $\eta$ in $H$ when $\lambda=0$, and hence, by Theorem 3, that the roots of $u_{1}(x, 0)$ and $u_{2}(x, 0)$ do not lie on $x_{1} x_{2}$. This means that the focal points $1^{\prime}$ and $2^{\prime}$ of $C_{1}$ and $C_{2}$ do not lie on the arc 12 of $E$. Furthermore the condition $u_{2} u_{1}^{\prime}$

* See Bliss, Mathematische Annalen, vol. 58 (1904), p. 70. 
$-u_{1} u_{2}^{\prime}>0$ implies that the derivative $d\left(u_{2} / u_{1}\right) / d x$ is less than or equal to zero, so that the equation $u_{1}=0$ can have no root $x_{1}^{\prime}>x_{2}$ preceding the first root $x_{2}^{\prime}$ of $u_{2}=0$. The points 1 and $1^{\prime}$ are therefore surely not separated by 2 and $2^{\prime}$ on the $\operatorname{arc} E$. This is the complete Jacobi condition as described in the reference of the footnote on the preceding page.

University of Chicago

\section{A CONNECTED AND REGULAR POINT SET WHICH CONTAINS NO ARC*}

BY R. L. MOORE

A point set is said to be connected im kleinen, $\uparrow$ or regular, at the point $P$ if, for every positive number $e$, there exists a positive number $d_{e}$ such that if $X$ is any point of $M$ at a distance from $P$ less than $d_{e}$ then $X$ and $P$ lie together in some connected $\ddagger$ subset of $M$ of diameter less than $e$. A point set which is regular (connected im kleinen) at every one of its points is said to be regular (connected im kleinen). The set $M$ is uniformly connected im kleinen if for each positive number $e$ there exists a positive number $d_{e}$ such that every two points of $M$ at a distance apart less than $d_{e}$ lie in a connected subset of $M$ of diameter less than $e$. If a point set $M$ is con-

* Presented to the Society, September 6, 1923.

$\dagger$ Cf. Hans Hahn, Ueber die allgemeinste ebene Punktmenge, die stetiges Bild einer Strecke ist, JAHresbericht DER Vereinigung, vol. 23 (1914), pp. 318-322. Also S. Mazurkiewicz, Sur les lignes de Jordan, Fundamenta Mathematicae, vol. 1 (1920), pp. 166-209. This conception, as applied to a simple closed curve, was used by Pia Nalli in the paper Sopra una definizioni di dominio piano limitato da una curva continua, senza punti multipli, Rendiconti di Palermo, vol. 32 (1911), pp. 391-401.

$\ddagger$ According to Hahn's formulation, $X$ and $P$ lie in a closed and connected subset of $M$ of diameter less than $e$. It has been customary with me to omit the stipulation that this subset should be closed. However, the set $M$ described below is connected im kleinen according to either definition. 